\title{
La investigación pedagógica formativa como estrategia pedagógica para desarrollar el potencial de aprendizaje y pensamiento científico
}

\author{
The formative pedagogical research as pedagogical strategy for developing the \\ learning potentiall and scientific thinking
}

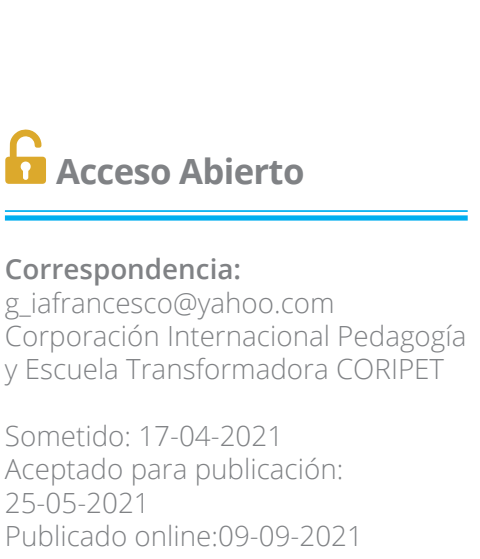

Palabras clave:

Calidad educativa; construcción del conocimiento; estrategias pedagógicas; investigación pedagógica formativa; procesos investigativos.

Key words:

Educational quality; knowledge construction; pedagogical strategies; formative pedagogical research; investigative processes.

\section{Citación:}

lafrancesco Villegas GM. La investigación pedagógica formativa como estrategia pedagógica para desarrollar el potencial de aprendizaje y pensamiento científico. Magna Scientia UCEVA 2021; 1 : 58-67. https://doi.org/10.54502/ msuceva.v1n1a9.
Giovanni Marcelo lafrancesco Villegas

\section{Resumen}

El objetivo de esta reflexión es establecer los lineamientos mínimos y orientaciones básicas para la elaboración y presentación de proyectos de Investigación Pedagógica Formativa (IPF). Se exponen las estrategias pedagógicas que facilitan los diversos procesos investigativos que apoyados en la IPF, forman nuevos investigadores. El mejorar la calidad educativa, los procesos de formación integral y de autoaprendizaje, supone intensificar la investigación en las Instituciones de Educación Superior (IES) y contribuir además con la práctica educativa y formativa. El aumentar la calidad de la actividad educativa e intensificar la investigación científica y tecnológica desde ella, supone también, una reconsideración de la investigación educativa y pedagógica, un cambio de óptica, tratando de formar a los investigadores actuales para que formen nuevos investigadores, con herramientas cognitivas pertinentes y con estrategias pedagógicas que aseguren el relevo generacional propio de las comunidades académicas e investigadoras.

\section{Abstract}

The aim of this reflection is to establish the minimum guidelines and basic orientations for the elaboration and presentation of Formative Pedagogical Research (FPR) projects. Some pedagogical strategies that facilitate the various investigative processes that, supported by FPR, form new researchers, are exposed. Improving educational quality, comprehensive training and self-learning processes, means intensifying research in Higher Education Institutions (HEI) and also contributing to educational and training practice. Increasing the quality of educational activity and intensifying scientific and technological research from it also supposes a reconsideration of educational and pedagogical research, a change of perspective, trying to train current researchers to train new researchers, with relevant cognitive tools and pedagogical strategies that ensure the generational renewal of the academic and research communities. 


\section{Introducción}

La investigación en educación y pedagogía tiene dos impactos centrales: i) producir conocimiento y comprensión sobre la educación al estudiar problemas, tales como los relacionados con la educación y la formación del ser humano en contextos claramente caracterizados o, los relacionados con los procesos de construcción del conocimiento y ii) producir nuevo saber en las ciencias y de las disciplinas del saber $\mathrm{y}$, de las construcciones conceptuales que en las mismas se promueven desde la formación de las múltiples inteligencias a través de la gestión y evaluación curricular en las instituciones educativas de todo carácter y nivel [1].

la Investigación Pedagógica Formativa (IPF), propicia las condiciones necesarias para la formación de nuevos investigadores [2], fomenta la creación de ambientes propicios para la investigación y el desarrollo científico y se caracteriza por lo siguiente: $i$ ) ser realizada por el educador mediador sobre sus educandos y sobre sus diferentes procesos de desarrollo, de forma específica o de forma integral; ii) ser operada en los espacios dinámicos de formación institucional y en las aulas de clase en los procesos de aprendizaje; iii) ser teórica, práctica, o teórico-práctica; $i v)$ tener carácter científico, pedagógico, o científico-pedagógico; $v$ ) fundamentarse en la observación sistemática, estructurada o no estructurada, denotativa o connotativa, experimental, cualitativa o cuantitativa, paramétrica o no paramétrica; vi) hacerse de forma descriptiva-interpretativa $\mathrm{o}$, analítica-argumentativa o, predictiva-propositiva $\mathrm{y}$, realizarse aplicando diseños metodológicos preexperimentales, cuasi-experimentales y experimentales con criterios de validez y confiabilidad, o con diseños metodológicos no experimentales; más descriptivos, interpretativos, críticos y analíticos con, criterios de credibilidad, transferibilidad y comprobabilidad [3].

Formar profesionales nuevos que den respuestas nuevas a las condiciones nuevas del continuo devenir, autogestionarios, proactivos, protagónicos, comprometidos, laboriosos, productivos, ingeniosos, creativos, innovadores, inventores, con pensamiento divergente y capacidad de solucionar problemas, solo se logra desde la investigación pedagógica que les permita desarrollar su potencial de aprendizaje y su pensamiento científico [4], con una formación humana y social, con inteligencia emocional, pero acompañada de unas habilidades mentales de alto nivel, que superen el conocimiento, su comprensión, su aplicación, su análisis y su síntesis, con la investigación, la caracterización y resolución de problemas, la creación de nuevos recursos, métodos, técnicas, procesos, programas y proyectos que mejoren la calidad de vida de las personas y de las comunidades de todo estrato social y respondan, con coherencia y pertinencia, a sus verdaderas necesidades [5].

La presente reflexión aborda el contexto de las estrategias pedagógicas que facilitan los diversos procesos investigativos que apoyados en la Investigación Pedagógica Formativa, propicie la formación de nuevos investigadores con pensamiento científico.

\section{La Investigación Pedagógica Formativa (IPF)}

Desde la perspectiva de la propuesta de Educación, Escuela y Pedagogía Transformadora (EEPT), el término investigación, significa: "ir tras los vestigios y consultar e indagar, con persistencia sistemática, un conocimiento deseado, definiendo campos, objetos, métodos, niveles, tipos, modalidades, estilos, enfoques y diseños metodológicos, con el propósito de contextualizar los problemas y, produciendo nuevo saber, intentar resolverlos, de forma provisional o de forma definitiva" [6].

Este término investigación, se aplica también a la educación, a la formación y a la pedagogía y, por este motivo, la Investigación Pedagógica Formativa -IPF-, se convierte en la estrategia más apropiada para producir el mejoramiento continuo y ayudar a la calidad y a la excelencia, en todas las instancias de la educación, de todo carácter y nivel y, en todas sus modalidades.

La IPF, de acuerdo con Iafrancesco Villegas [2], se define como el proceso creativo, constructivo, riguroso, objetivo, controlado y crítico, que sobre la base del conocimiento educativo y pedagógico disponible, busca resolver problemas, produciendo conocimiento y estrategias, para mejorar los procesos formativos y sus resultados, con coherencia y con pertinencia social y cultural [2]. 
Es necesario que en las Instituciones de Educación Superior (IES) se creen programas y líneas de investigación, articuladas entre sí, a través de núcleos problemáticos y temáticos, que se desarrollen mediante proyectos y trabajos de investigación, de forma holística. La estructuración por núcleos problemáticos y temáticos, puesta en marcha, desarrollo, implementación y consolidación de los programas, las líneas, los proyectos y los trabajos de investigación, dependen, en gran medida, de la formación de los educadores mediadores investigadores, de su vocación educativa, formativa y científica, de su profesión docente, de su trabajo permanente con la formación de las nuevas generaciones $\mathrm{y}$, del empeño diario por desarrollar excelentes procesos de enseñanza-aprendizaje, sin dejar de lado, claro está, las responsabilidades políticas de los Estados frente a la educación y, los recursos institucionales relacionados con el servicio de la investigación dentro de este proceso investigativo educativo, formativo y pedagógico [6].

\section{Las funciones cognitivas}

Para poder desarrollar inteligentemente los requisitos exigidos en un proyecto de investigación, no solo basta con saber las partes que comprenden un proyecto (éstas por lo general, no cambian), depende de la complejidad del problema de investigación y de los métodos empleados para poder resolverlo [2]; en este escenario, se logran evidenciar estructuras cognitivas en el tipo de inteligencia del investigador (lógico-matemática, naturalística o científica, espacio-temporal, lingüística, kinestésica, musical, ético-moral y emocional), en el desarrollo de sus funciones cognitivas, de sus procesos de pensamiento, de sus operaciones intelectivas y de sus competencias cognitivas, con las cuales puede definir el alcance del proyecto de investigación e incluso, las limitaciones que éste tendría por la carencia de recursos cognitivos para mejorar la calidad de solución a los siguientes problemas de funcionamiento cognitivo: $i$ ) previo al proceso investigativo; $i$ ) durante la elaboración conceptual; iii) en el manejo de los diseños metodológicos de investigación y $i v$ ) durante la exposición, socialización o sustentación de los proyectos desarrollados.

Algunas de las deficiencias más comunes de funcionamiento cognitivo que tienen los educandos que no han sido formados en investigación (figura 1) son: i) la percepción fragmentada y/o distorsionada; ii) la carencia de recursos verbales o de habilidades lingüísticas previas; iii) la impulsividad; iv) la inadecuada orientación espacial y/o temporal; v) la dificultad para conservar lo esencial de lo observado y percibido; vi) la imprecisión e inexactitud en la recolección de información y en la organización de la misma (figura 1).

Algunas de las deficiencias más comunes de funcionamiento cognitivo que tienen los educandos durante la elaboración conceptual y el manejo de los diseños metodológicos de investigación (figura 1) son: $i)$ el no poder establecer relaciones entre datos o informaciones; ii) la imposibilidad de caracterizar; iii) la imposibilidad de seleccionar datos relevantes e importantes; $i v$ ) la dificultad para hacer comparaciones; v) la estrechez del campo mental; vi) la dificultad para globalizar y generalizar; vii) la dificultad para establecer relaciones lógicas causa-efecto; viii) la imposibilidad para evaluar las propias actitudes y comportamientos; $i x$ ) la dificultad para formular hipótesis y hacer inferencias; x) la imposibilidad de demostrar, comprobar o verificar hipótesis; xi) la dificultad para planear, programar y llevar a cabo lo planeado y programado; xii) la rigidez para trabajar intelectualmente desde solamente una categoría cognitiva; xiii) la dificultad para construir síntesis conceptuales y sacar conclusiones; xiv) la imposibilidad de establecer relaciones virtuales (figura 1). 


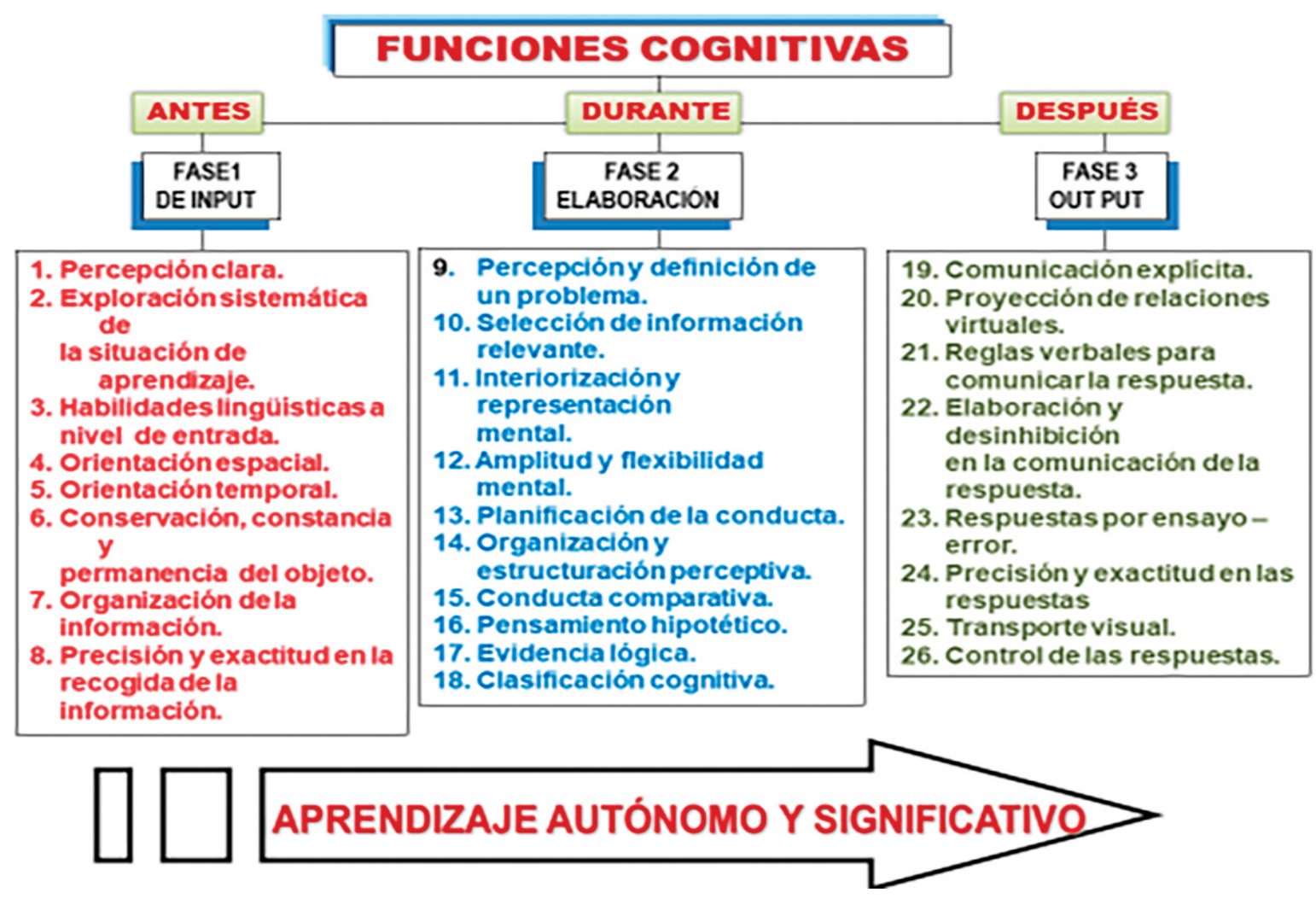

Figura 1. Funciones cognitivas en las fases de input (antes de aprender), de elaboración (mientras se aprende) y de output (después de aprender)

\section{Operaciones intelectivas}

El desarrollar las 26 funciones cognitivas expuestas en la figura 1, permite a los educandos formados como investigadores, desempeñar las operaciones intelectivas que se enseñan en la abla 1 . Todos estos procesos cognitivos, si son deficientes, generan problemas de aprendizaje, como también actitudinales frente al conocimiento y de incertidumbre durante los trabajos prácticos que se realizan. La investigación, con su organización lógica y coherente, con el proceso de indagación que demanda la caracterización de problemas y su resolución, denota la necesidad que implica formular objetivos y estrategias para alcanzarlos, con la necesidad de una revisión rigurosa, coherente y apropiada, con la necesidad de utilizar métodos pertinentes y desarrollar estrategias inteligentes para ser eficiente, eficaz y efectivo en la forma de resolver los problemas; termina convirtiéndose en el mejor espacio y escenario de formación cognitiva, desde la misma práctica investigativa $\mathrm{y}$, por lo tanto, es un excelente recurso pedagógico para mejorar los procesos de pensamiento de los educandos, en situaciones concretas, a manera de estudios interpretativos de casos (tabla 1).

Esta es la función de la Investigación Pedagógica Formativa (IPF), se aprende investigando y se investiga aprendiendo, puesto que siempre el resultado será diferente [2]. Por eso, los expertos son inversamente proporcionales a los científicos, debido a que, quien utiliza el mismo método para todo, refleja que solo conoce el método y que desvirtúa los problemas que no puede resolver con el único método que conoce. El científico investiga, no solo desde la teoría, las leyes y los principios, sino desde los procesos y métodos que debe emplear, de forma específica, en cada situación problema [5].

\section{La alternativa}

Desde la perspectiva cognitiva y desde la visión dada sobre las funciones cognitivas y las operaciones intelectivas que pueden expresarse en las diferentes inteligencias, se demuestra que la investigación permite el desarrollo de la cognición [7]. 


\section{No. Operación intelectiva}

\section{Definición y/o alcance}

1 Identificación Equivale al reconocimiento de una realidad por sus características globales recogidas en un término que la define y la describe

2 Diferenciación Equivale al reconocimiento de algo por sus características, distinguiendo las que son esenciales de las que son irrelevantes en cada situación de la que dependen

3 Representación Consiste en la interiorización de características de un objeto de conocimiento, sea éste concreto o mental abstracto. No es la fotografía mental del objeto, sino la representación de los rasgos esenciales que permiten definirlo como tal

4 Transformación Equivale a la actitud cognitiva a través de la cual podemos modificar o combinar características de mental un objeto, o de varios objetos, para producir representaciones de mayor grado de abstracción o de complejidad

5 Comparación Equivale a la operación mental o intelectiva en la que, partiendo de una percepción clara y estable, se estudian las semejanzas y diferencias entre objetos, o hechos, atendiendo a sus características

6 Clasificación Equivale a la reunión de grupos de elementos de acuerdo con atributos definitorios, basada en criterios arbitrarios, naturales o artificiales, según se realicen sobre cosas, hechos o conceptos

7 Codificación- Consiste en establecer símbolos o interpretarlos, de tal modo, que no dejen lugar a la ambigüedad. decodificación Esta operación mental permite dar amplitud a los términos y símbolos, a medida que aumenta el nivel de abstracción

8 Proyección de Consiste en la proyección mental de estímulos externos, percibidos en forma de unidades organizadas relaciones de información y, que luego proyectamos mentalmente ante estímulos semejantes, mediante imágenes, virtuales ocupando con ellas un lugar en el espacio

$9 \quad$ Análisis y la síntesis

Como formas de percibir la realidad, de manera deductiva e inductiva respectivamente, del todo a las partes o de las partes al todo, de lo general a lo particular o de lo particular a lo general, que permiten descomponer un todo en sus elementos estructurales constitutivos (análisis) y relacionándolos entre sí y con el todo (síntesis), para generar inferencias: Los análisis permiten y facilitan la síntesis

10 Inferencia lógica

Consiste en la capacidad para realizar deducciones y crear nueva información a partir de los datos percibidos

11 Razonamiento Es equivalente al razonamiento proporcional, basado en los argumentos inductivos, dentro de un analógico ámbito "tolerante extenso", que permite, dados tres términos de una proporción, determinar el cuarto por deducción de la semejanza. Por lo general, el razonamiento analógico no se acepta como argumento demostrativo, pero sí como descubrimiento y como muestra de convicción.

12 Razonamiento Equivalente a la capacidad mental de realizar inferencias y predicciones de situaciones o hechos a hipotético partir de los ya conocidos y, de las leyes que los regulan y relacionan

13 Razonamiento Equivale a la capacidad mental que le permite a una persona llegar a una verdad lógica, surgida ésta, silogístico de la propia construcción mental, mediante el ejercicio del pensamiento lógico y el desarrollo de la capacidad de construir modelos mentales, de situaciones o escenarios dados, ayudándose de las leyes de la lógica

14 Razonamiento Es equivalente a la capacidad de establecer nuevas relaciones sobre lo que ya se conoce, de modo que divergente se llegue a productos nuevos, en forma de ideas, conceptos, realizaciones o fantasías. Está asociado a lo inusual, a la flexibilidad mental, al ingenio, a la creatividad, a la capacidad de innovación e invención, en contraposición al razonamiento convergente que se genera del dominio riguroso de los datos, de la precisión, de la exactitud, del rigor científico y de lo convencional y ya establecido

15 Razonamiento Puede considerarse como una representación de una representación de acciones posibles, propia del lógico pensamiento formal, del buen pensar, de la organización del pensamiento que llega a la verdad lógica gracias a diversas formas de razonamiento, entre ellas, el inferencial, el hipotético y el silogístico 
Solo hay que ir de lo sencillo a lo complejo, de forma secuencial, organizada, acumulativa, que permita el desarrollo progresivo para asegurar el mejoramiento continuo, el desarrollo del potencial de aprendizaje y la capacidad transformadora de quien, frente a los problemas, los resuelve o, por lo menos, plantea posibilidades de resolverlos con pensamiento pre, cuasi o investigativo (antes, mientras y después de aprender). La organización del anteproyecto de investigación, su desarrollo teórico y práctico, su aplicabilidad en situaciones problema, su validación y sus propuestas alternativas para la resolución de los problemas; les permite a los educandos que aprenden a investigar, desarrollar las competencias cognitivas básicas y los procesos de pensamiento científico en cuanto a: $i$ ) la mecanización; ii) la concreción; iii) la configuración; iv) la abstracción $\mathrm{y} v$ ) la lógica y la formalización (ver figura 2).

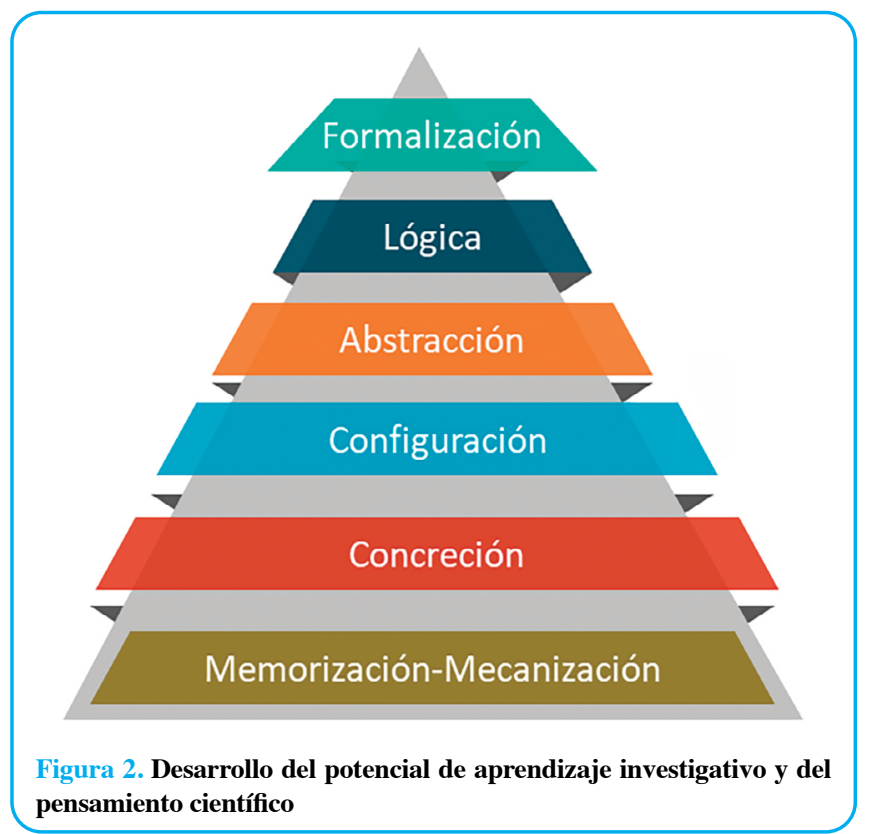

Estos procesos se desarrollan evolutivamente, partiendo de lo simple a lo complejo, de estructuras cognitivas menos consistentes a estructuras cognitivas más consistentes y de forma secuencial, progresiva y acumulativa; los cuales se asocian a la madurez mental y corresponden a los niveles de memoria mecánica, configurativa y lógica y, a los niveles de pensamiento concreto, abstracto y formal [1].
La Investigación Pedagógica Formativa (IPF), se convierte entonces, en un trabajo de formación del pensamiento científico y del desarrollo del potencial de aprendizaje, teniendo en cuenta la madurez de los procesos cognitivos, se convierte también en una excelente estrategia para formar nuevos investigadores y para generar nuevos procesos de transformación en las Vicerrectorías Académicas y de Investigación en las Universidades, transformando el currículo de los programas Académicos, sus planes de estudio, las unidades didácticas desarrolladas en ellos para alcanzar nuevos logros actitudinales, conceptuales y procedimentales que faciliten el desarrollo cognitivo [2].

Estos nuevos profesionales, formados desde esta perspectiva investigativa, se caracterizarán por sus expectativas, intereses, motivación y dedicación a la investigación; con excelente desarrollo de sus procesos de pensamiento, con habilidades mentales, funciones cognitivas y operaciones intelectivas de alto nivel que les permitirá una excelente estructuración y construcción conceptual expresadas en sus competencias cognitivas básicas y sus excelentes desempeños investigativos, evidenciados éstos, en sus hábitos, habilidades y destrezas en el manejo de métodos, técnicas, procesos, procedimientos $\mathrm{y}$ estrategias para resolver problemas con eficiencia, eficacia y efectividad, siendo excelentes en su forma de ser (competencias antropológicas), de sentir (competencias afectivas), de actuar (competencias éticas y morales), de vivir (competencias axiológicas y espirituales) y de convivir (competencias sociales y ciudadanas) (figura 3); lo que los hará ser promotores de paz, vida y convivencia; como también, excelentes en su forma de saber (competencias académicas y científicas), de saber hacer (competencias laborales y ocupacionales), de pensar (competencias cognitivas e intelectivas), de aprender (competencias investigativas y tecnológicas) y de emprender (competencias de liderazgo y emprendimiento), lo que los hará ser promotores de éxito y de los desarrollos sostenible y sustentable, siendo parte de la solución y no del problema (ver figura 3). 


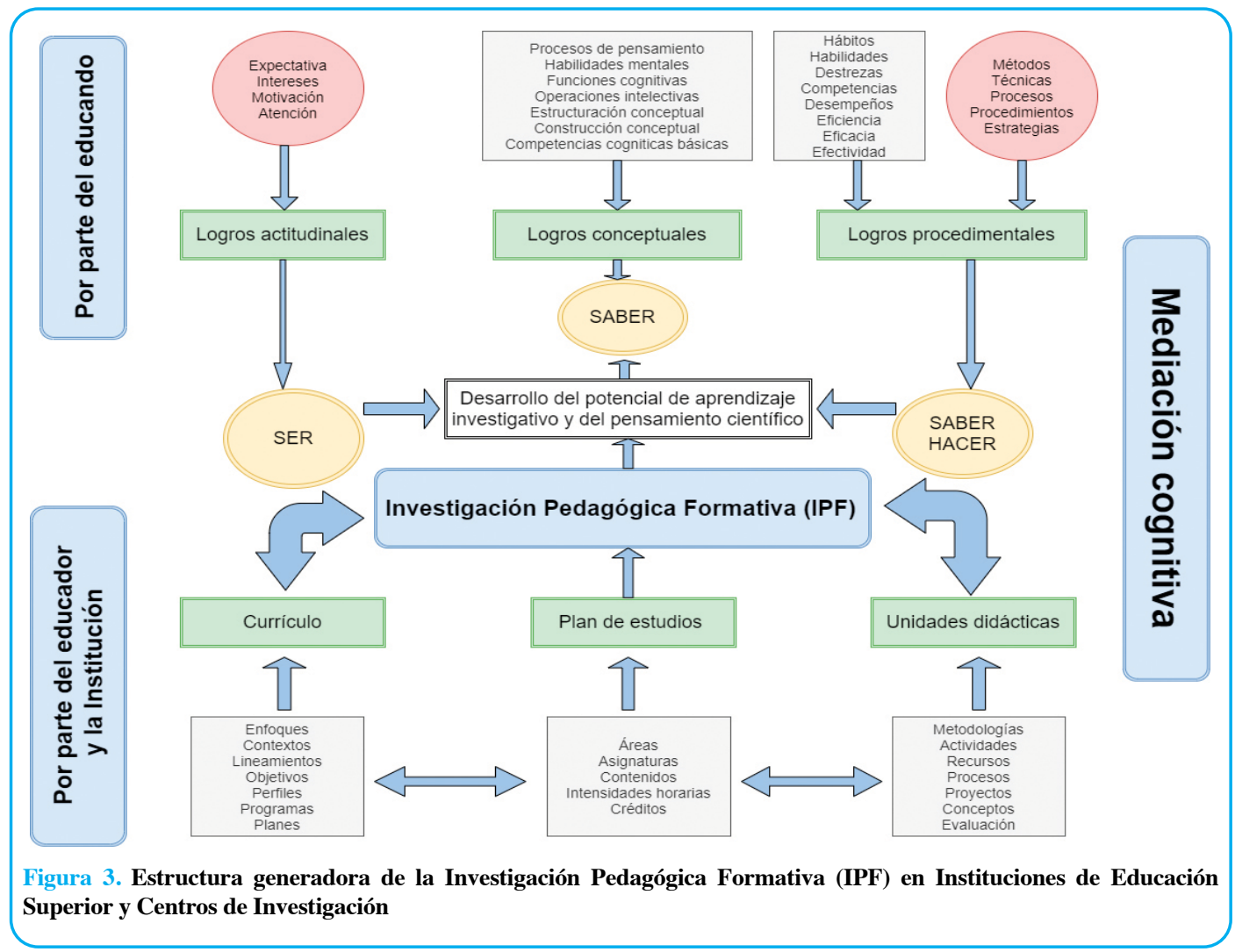

\section{Propuesta de estrategia cognitiva mediada para facilitar la estructuración y evaluación de proyectos de investigación desde la perspectiva (IPF)}

Esta estrategia no solo sirve para presentar los proyectos de investigación como se sugiere presentarlos, especialmente sirve para estructurarlos caracterizando muy bien los elementos que integran el proyecto, las funciones de estos elementos dentro del mismo, estableciendo las correctas relaciones entre ellos y con la estructura implícita, lo que le permite dar coherencia al proyecto de investigación y, que a lo largo de su organización, elemento por elemento, función por función y relación por relación, les permite asegurar su pertinencia.

\section{Características deseables en un proyecto de investigación}

Un buen diseño de proyecto de investigación debe poseer las siguientes características: $i$ ) Es válido, si es pertinente y está adecuado a los fines requeridos por la ciencia, por la pedagogía, por la Institución y por la sociedad; ii) económico, si propone las soluciones que exigen menores esfuerzos, tiempos y financiamientos (eficiencia, eficacia, efectividad) y en lo posible, si es autofinanciable; iii) flexible, si permite revisiones y ajustes durante la etapa de desarrollo sin perder de vista los objetivos propuestos y sin restarle calidad y iv) formalmente elaborado, si facilita su interpretación y comprensión, si es preciso en sus afirmaciones y, si es lo más conciso posible.

\section{Recomendaciones estratégicas para la estructuración y evaluación de los proyectos de investigación}

Para facilitar, no solo la organización y presentación del proyecto, sino también su estructuración coherente y su autoevaluación, para tener certeza de que ya está listo para ser presentado a quienes lo evalúan, lo aprueban o lo financian, sean universidades o, entidades promotoras o financiadoras, los educadores mediadores investigadores, deben tener en cuenta las siguientes recomendaciones estratégicas: 
- El título (y el subtítulo si lo hay), deben reflejar claramente: el problema por resolver y la estrategia utilizada para resolverlo. Debe entonces, de forma explícita, presentar las variables dependiente $\mathrm{e}$ independiente $\mathrm{y}$, de forma implícita, la hipótesis. También debe reflejar el paradigma, el enfoque, la escuela de pensamiento, el autor o el modelo, cuando éstos, o alguno de estos, se den y exista marco teórico.

- El título debe relacionarse con el objetivo general del trabajo y solo diferenciarse de él, en la formulación del verbo en infinitivo y la descripción, sintética, del contexto o la población investigada.

- Para caracterizar muy bien el problema de investigación, es necesario realizar, de forma deductiva, de lo general a lo particular los siguientes pasos: descripción del problema, delimitación del problema, definición del problema, planteamiento del problema y formulación del problema.

- La descripción y la delimitación del problema deben relacionarse con el título del proyecto, en especial, con la variable dependiente. La definición y el planteamiento del problema deben relacionarse con el subtítulo del problema, en especial, con la variable independiente. La formulación del problema debe relacionarse con la hipótesis de trabajo y las variables explícitas en ella.

- Los antecedentes empíricos, si éstos existen, deben relacionarse con el marco contextual y el marco situacional. Los antecedentes bibliográficos y documentales, deben relacionarse con el marco teórico y el marco conceptual.

- La justificación debe relacionarse con la caracterización del problema, pero también con el valor agregado del proyecto (bondades, beneficios, aportes, impacto), el cual puede verse reflejado en el objetivo general, en algunos objetivos específicos, pero, fundamentalmente, al terminar el proyecto, con las conclusiones y las limitaciones superadas.

- El objetivo general se relación con el título del proyecto, pero los objetivos específicos, sin descontextualizarse de él, tienen relación, fundamentalmente, con los pasos del protocolo de la investigación (diseño metodológico) o las fases del procedimiento, siguiendo el estricto orden de ejecución de ellas, de forma secuencial, acumulativa y progresiva, a través de las actividades propuestas en el cronograma.

- La factibilidad tiene relación con los recursos sugeridos y presentados en el proyecto y con las limitaciones que puedan presentarse en su uso, manejo o consecución. Las limitaciones tienen relación con el alcance del proyecto, debido a la factibilidad del mismo y, desde ellas, con las sugerencias y recomendaciones que se harán, a otros investigadores, al término del trabajo de investigación.

- El marco de referencia puede contener varios marcos, todos, o algunos de ellos o, mínimo uno de ellos. El marco teórico es el referente del marco conceptual y, los dos, ayudan a aclarar el problema y permiten presentar los fundamentos teóricos para ayudar a resolverlo. El marco contextual es el referente del marco situacional y, los dos, permiten caracterizar las condiciones en las cuales se da el problema por resolver, desde la investigación. El marco situacional, define en parte, las limitaciones para llevar a cabo el proyecto.

- Las hipótesis y las variables tienen relación con la forma como se definirá el diseño metodológico y la forma como se ejecutará el procedimiento de investigación, de acuerdo con la naturaleza de la misma.

- El diseño metodológico aporta la naturaleza de la investigación, el tipo de procedimientos empleados, la población y la muestra, los instrumentos de recolección de información, el tratamiento estadístico de las variables, la tabulación, graficación y análisis de los datos y, de estos elementos, dependen claramente las conclusiones del trabajo, su validez y su confiabilidad.

- Los protocolos o procedimientos utilizados en el diseño metodológico, las etapas, las fases y los pasos sugeridos en ellos, se relacionan, con los objetivos específicos, aportando indicadores de logro del proceso desarrollado en búsqueda de alcanzar el objetivo general; por eso, los objetivos deben ser redactados de acuerdo con el procedimiento 
(etapas, fases, pasos), en el mismo orden, siguiendo la misma secuencia, de forma progresiva.

- Los protocolos o procedimientos utilizados en el diseño metodológico, también se relacionan con el plan operativo, con la administración del proyecto y con el cronograma de actividades, ya que desde el desarrollo de las etapas, las fases y los pasos del mismo, se organizan las secuencias del desarrollo de las actividades y se definen los tiempos para realizarlas.

- El cronograma de actividades evidencia que el procedimiento se llevará a cabo y que los objetivos específicos se cumplirán; es por esto que el plan operativo debe organizarse relacionando, estructuralmente: objetivos específicos, diseño metodológico, procedimiento y cronograma.

- La bibliografía citada se relaciona con el marco de referencia, especialmente con el marco teórico y el marco conceptual, al igual que con el glosario (o palabras clave) para la definición de términos y con los antecedentes bibliográficos; pero la bibliografía consultada se relaciona, con elementos constitutivos del proyecto, en especial, con el problema, con la justificación y con los antecedentes bibliográficos.

- Los anexos ayudan a, que quien lea, estudie, evalúe y apruebe el proyecto, tenga certeza, evidencia y confiabilidad sobre la realización del mismo y de la investigación, por cuanto en los anexos se encuentran todos los soportes, para demostrar, prospectivamente, que el trabajo se podrá realizar, pues en los anexos están las cartas aprobatorias del proyecto, financiadoras o patrocinadoras del mismo, los convenios, el apoyo del director del proyecto, de los asesores, de los coinvestigadores, de los pares académicos y de los promotores de estos proyectos. Por estos motivos, los anexos se relacionan con la factibilidad de la investigación, debido a que la aseguran.

- También en los anexos, deben incluirse los instrumentos utilizados en el diseño metodológico para recoger la información y los datos, con las variables y con los tratamientos estadísticos, representados en tablas y gráficas, con mapas si los hay y demás elementos que considere, por el carácter de la investigación, ser presentados.

- Tanto en la bibliografía, como en los anexos, se deben relacionar todas las fuentes de consulta utilizando el estilo bibliográfico al que se ajuste la Institución y lo defina en los lineamientos de presentación de los proyectos de investigación (APA, Vancouver, IEEE, etc.), que a la fecha de realización del proyecto estén vigentes.

\section{Conclusión}

Establecer las relaciones entre los distintos elementos constitutivos del proyecto de investigación (título, problema, antecedentes, justificación, objetivos general y específicos, factibilidad, limitaciones, marco de referencia, hipótesis y variables, diseño metodológico, plan operativo y administrativo, bibliografía y anexos), mediante el mapa cognitivo estructural y evaluativo implícito en la Institución, le permiten al educador mediador investigador: $i$ ) estructurar el proyecto de forma inteligente; $i$ ) evaluar la coherencia del proyecto de Investigación Pedagógica Formativa (IPF) antes de iniciar el desarrollo del mismo; iii) sistematizar previamente el trabajo con coherencia y pertinencia; $i v$ ) prever las limitaciones; $v$ ) crear nuevas alternativas; $v i$ ) flexibilizar los recursos y los tiempos y, vii) asegurarse, con evidencia lógica y pensamiento estructural prospectivo, que todo lo previsto se podrá realizar. Todo esto es lo que hace que un investigador sea eficiente, eficaz, efectivo, pertinente, coherente y competente.

\section{Consentimiento para publicación}

El autor leyó y aprobó la versión final del manuscrito.

\section{Conflicto de interés}

El autor declara no tener ningún tipo de conflicto de interés con la publicación. 


\section{Perfil de autoría}

Giovanni Marcelo Iafrancesco Villegas

Es una autoridad mundial en pedagogía, $\mathrm{PhD}$ en Filosofía de la Educación en Newport University, USA; PhD en Educación de la Universidad Internacional Euroamericana de Valencia, España; Mag. en Docencia Universitaria de la Universidad de La Salle, Bogotá-Colombia; Mag. en Estudios Profesionales del Instituto Latinoamericano de Estudios Profesionales

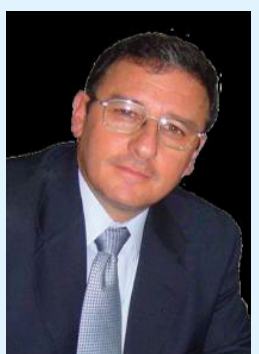

(ILAEP) de México; Mag. en Estudios Estratégicos del Instituto de Estudios Estratégicos (IDEE) de Montevideo-Uruguay. Galardonado con 106 distinciones y premios honoríficos en 14 países de Iberoamérica; entre ellos, 9 Doctorados y 7 Maestrías Honoris Causa. Es actualmente, director de la Corporación Internacional Pedagogía y Escuela Transformadora (CORIPET); asesor, consultor y conferencista a nivel nacional e internacional. Profesor invitado de postgrados (Doctorados, Maestrías y Especializaciones) en universidades colombianas y extranjeras de países de Iberoamérica. Es además consultor del Programa de Naciones Unidas para el Desarrollo (PNUD) de la Organización de Naciones Unidas (ONU) en Panamá; miembro fundador y de número de la Academia Colombiana de Pedagogía y Educación; creador y director de la propuesta de Educación, Escuela y Pedagogía Transformadora (EEPT) para América Latina, posicionada hoy en 27 países; autor de textos didácticos en Ciencias Naturales para Colombia, Nicaragua y El Salvador y otros textos académico-científicos.

\section{Referencias}

[1] Iafrancesco Villegas GM. La evaluación en el aula de una Escuela Transformadora: contexto, concepto, objetivos, principios, enfoques, tipos, modelos y estrategias. 2nd ed. Bogotá, Colombia: CORIPET EDITORIAL. Corporación Internacional Pedagogía y Escuela Transformadora CORIPET Ltda; 2017.
[2] Iafrancesco Villegas GM. La Investigación Pedagógica Formativa: contextos, fundamentos, diseños metodológicos, técnicas, instrumentos y estructuración de proyectos. 1st ed. Bogotá, Colombia: CORIPET EDITORIAL. Corporación Internacional Pedagogía y Escuela Transformadora CORIPET Ltda; 2013.

[3] Iafrancesco Villegas GM. La administración pedagógica en una Escuela Transformadora: concepto, principios, funciones, estilos políticas y estrategias de planeación, organización, dirección y control. 1st ed. Bogotá, Colombia: CORIPET EDITORIAL. Corporación Internacional Pedagogía y Escuela Transformadora CORIPET Ltda; 2013.

[4] Iafrancesco Villegas GM. Aprendizaje autónomo y cognición: fundamentos y estrategias didácticas para el desarrollo del potencial de aprendizaje y del pensamiento. 3rd ed. Bogotá, Colombia: CORIPET EDITORIAL. Corporación Internacional Pedagogía y Escuela Transformadora CORIPET Ltda; 2017.

[5] Iafrancesco Villegas GM. Las transformaciones de las Prácticas Pedagógicas: contextos, métodos, paradigmas, modelos, estrategias y políticas. 2nd ed. Bogotá, Colombia: CORIPET EDITORIAL. Corporación Internacional Pedagogía y Escuela Transformadora CORIPET Ltda; 2015.

[6] Iafrancesco Villegas GM. La gestión y la evaluación curricular en una Escuela Transformadora: Contextos, enfoques, diseños, concepto, estructura, modelo holístico, objetivo, planeamiento y protocolo. 1st ed. Bogotá, Colombia: CORIPET EDITORIAL. Corporación Internacional Pedagogía y Escuela Transformadora CORIPET Ltda; 2013.

[7] Iafrancesco Villegas GM. Modelo Pedagógico Holístico Transformador: fundamentos, dimensiones, programas y proyectos en la Escuela Transformadora. 1st ed. Bogotá, Colombia: CORIPET EDITORIAL. Corporación Internacional Pedagogía y Escuela Transformadora CORIPET Ltda; 2015. 